\title{
The association of economic and cultural capital with the NEET rate: differential geographical and temporal patterns
}

Enrico Ripamonti ${ }^{1,2^{*}}$ (1) and Stefano Barberis ${ }^{3,4}$

\begin{abstract}
Using data from 103 Italian provinces, we investigated the relationship between local/regional development, and NEET. We constructed an indicator of cultural capital and another of economic capital and we studied their relation with the NEET rate. Covariance Structure Analysis with Generalized Least Squares estimation was employed, considering a three time-points retrospective model. Results indicate a consistent protective effect of the economic capital on the NEET rate, both in the short run (2 years) and in the medium run (10 years). However, this effect has been obtained in the Central provinces (at 2 and 10 years) and Southern provinces (at 10 years), but not in the Northern provinces. A mediation analysis indicated that, historically, the cultural capital may partly mediate the effect of the economic capital. We did not detect a significant direct effect of the cultural capital on the NEET rate, which is strongly mediated by the action of the economic capital. Together, these results denote that the economic capital is a strong predictor of NEET, but not in very competitive economic areas.
\end{abstract}

Keywords: Cultural capital, Economic capital, NEET, School dropout, Southern European welfare

\section{Introduction}

The individual trajectories of adolescent development, including educational attainment and participation in the labor market, should be studied in conjunction with the role of macro-level determinants, which may play a top-down effect on teenagers' behavior and interact with micro-level variables. This paper specifically investigates the role of economic and cultural capital on young people who are neither in employment nor in education or training (NEET)s. While being NEET reflects a multifactorial phenomenon, whose main determinants have been partially highlighted, the literature has paid less attention to the role of the economic capital and of the cultural wherewithal offered by a geographical area, as originally operationalized by Di Maggio and Mohr (1985). Herein

\footnotetext{
*Correspondence: enrico.ripamonti@unimib.it

${ }^{1}$ Milan Center for Neuroscience, University of Milan-Bicocca, Piazza dell'Ateneo Nuovo 1, 20126 Milan, Italy

Full list of author information is available at the end of the article
}

we will investigate these issues using national retrospective data.

Contemporary European societies are, in general, characterized by advanced and knowledge-based economies, as well as by strong welfare systems and social services. However, there is also high heterogeneity across and within countries (Mayer 2004), which makes important the study of macro-level variables for a deep understanding of social change and of youth transition to adulthood (Buchmann and Kriesi 2011). It is particularly worth placing the study of NEET in this high-level framework, since this may provide policy makers with a comprehensive view on the risk factors involved in this phenomenon. Indeed, governmental and international agencies, in particular the EU and its institutions, have long targeted this group of young people for intervention and monitoring. Such interest reflects the general objectives of the Lisbon strategy, ratified in 2000 by all Member States. However, on current state of research, there is large heterogeneity 


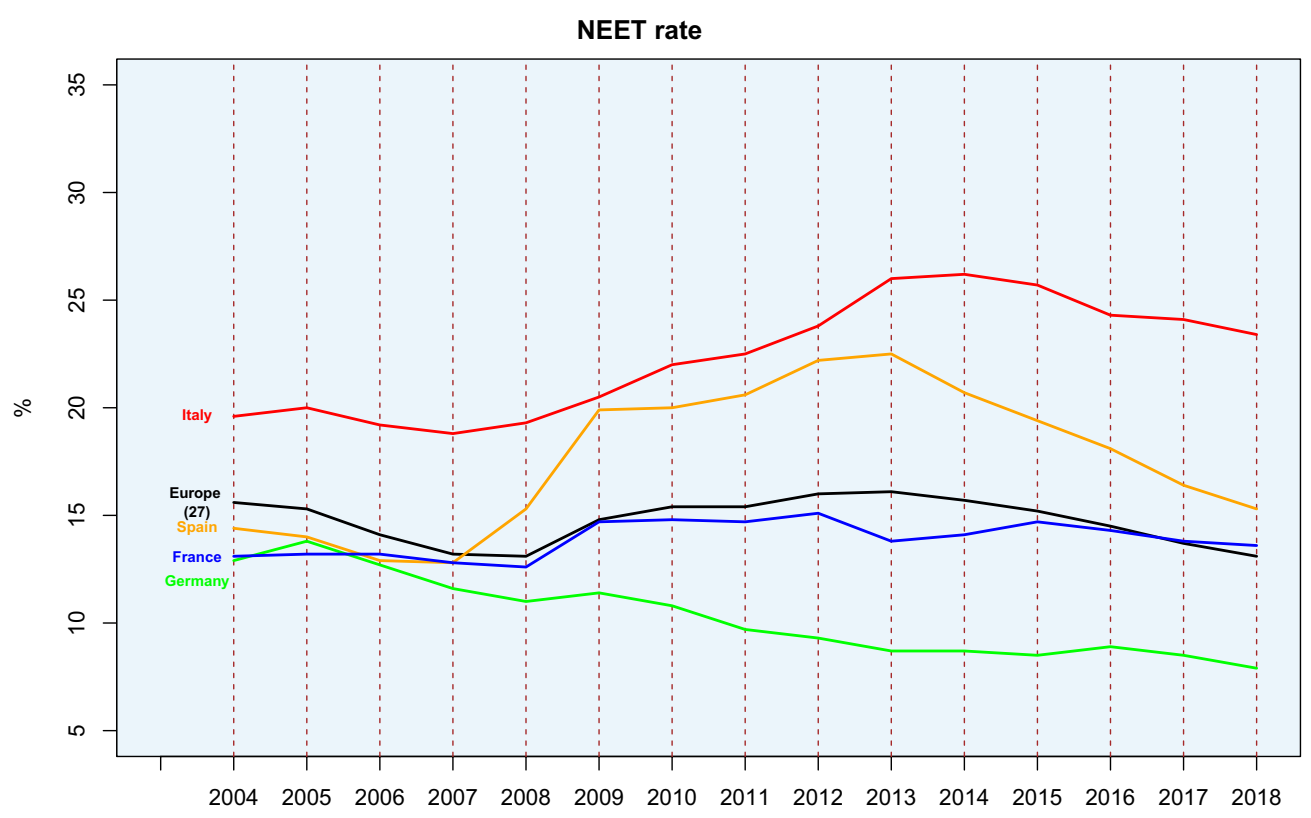

Fig. 1 Time series indicators of the NEET rate in the 27 EU countries, and, nationally, in France, Italy, Germany, and Spain

in methods and models followed in such interventions, without a clear and long-term strategy.

\subsection{NEETs in contemporary European societies \\ 1.1.1 Definition of NEET}

The concept of NEET refers to young people who, since are not working and, at the same time, are not enrolled in school or other formative activities, are not accumulating human capital (Eurofound 2012). Thus, while correlated to the construct of school dropout (Ripamonti 2018), NEETs reflect a more radical and extended form of vulnerability and social exclusion. The acronym NEET was firstly used in the United Kingdom in the 1980s, but became popular worldwide starting from 2010, when the European Union (EU) adopted the NEET rate as an official indicator to assess the youth conditions across EU countries. In Fig. 1 we show the time series of the NEET indicator for the $27 \mathrm{EU}$ countries, and, specifically, for Italy, Spain, France, and Germany. Further graphs and illustrations are presented in Additional file 1.

\subsubsection{The youth unemployment rate and the NEET rate}

The youth unemployment rate (u \%) can be defined (e.g., in the Labor Force Survey) as the ratio between the number of unemployed young people (\#U e.g., in the age range 15-24) and the total number of people in the same age range that make up the labor force $(\# \mathrm{~L}=\# \mathrm{U}+\# \mathrm{E}$, where $\mathrm{E}$ indicates the employed), namely (Blanchard et al. 2017):

$$
\mathrm{u} \%=\frac{\# \mathrm{U}}{\# \mathrm{~L}} * 100
$$

To be included in the definition of \#U a person must not have a job, but, at the same time should be actively searching for a job (otherwise he/she would be classified as out of L). While simple in principle, there is debate in the literature on the measurement of the labor force (Battistin et al. 2007) and of the unemployment rate (Jones and Riddell 1999). The NEET rate has a slightly more complicated definition. According to international institutions (e.g., the International Labor Organization or Eurostat), the NEET rate (n\%) can be defined as the percentage of the population of a given age, group or sex who is not employed and not involved in further education or training (Elder 2015):

$$
\mathrm{n} \%=\frac{(\# \mathrm{Y}-(\# \mathrm{YE}+\# \mathrm{YT}))}{\# \mathrm{Y}} * 100
$$

where \#Y indicates the number of youths, \#YE the number of youths in employment, \#YT the number of youths not in employment, but who are in education or training. ${ }^{1}$ It is worth placing the discussion on NEET and unemployed youth in the larger framework of the theory of human capital (Becker 1962). Educational achievement is indeed a proxy of human capital, which affects

\footnotetext{
${ }^{1}$ At the numerator, where subtracting from \# $\mathrm{Y}$, young people that are both in employment and in education or training simultaneously should not be double counted.
} 
the economic capital and the economic development (Barro 1991). In the 2000s the economic theory underlined the importance of non-cognitive skills (e.g., motivation, leadership, initiative, goal-oriented attitude) in the development of human capital (Heckman and Rubinstein 2001). To increasing levels of human capital correspond increased salaries, and the differential in real wages between skilled and unskilled workers, at least in the US, has enlarged from 1970 to 2005 (Autor et al. 2008). In addition, a past history of absence of high educational level and unemployment may also protect teenagers with respect to the probability of future unemployment and transition through the NEET status (Narendranathan and Elias 1993). Young people with low levels of education have relevant difficulties to entry in the labor market (Martin 2009). In Southern Europe, young people of all educational levels may experience difficulties in finding a job and career instability (Brzinsky-Fay 2007).

\subsubsection{NEET: A blurred concept}

In general, NEETs constitute a very heterogeneous group of individuals, who, however, share an increased risk of social and labor market exclusion. The very concept of NEET has long been criticized, since it would denote a too general as well as fragmented category, not adequate to represent the problems of young people in transition to adulthood (Furlong 2006). Heterogeneity refers to the fact that this category could virtually include, at the same time, young people who are not employed and not at school but are proactively searching for an occupation, as well as people virtually stable in their status, without putting in action proper efforts to ameliorate their condition. In the former group there are NEETs looking for their first job (having completed or not the school with a certificate), and long-term unemployed young people. In the latter group there are those who, as being long-term unemployed people, have been discouraged to search for an occupation, as well as young people who are temporarily in the NEET condition (e.g., to take care of children or parents). While, in general, the label "NEET" may capture young people at risk of marginalization in contemporary societies, there does not exist a shared operational definition underpinning this concept. In fact, scholars have defined the NEET phenomenon in very different ways and with reference to young people of different ages (Yates and Payne 2006). If we consider NEETs as young people aged 15-29, such definition would cover three quite different developmental phases, namely late adolescence (15-17), emerging adulthood (18-24), early adulthood (25-29) (Arnett 2006).

\subsubsection{Individual factors related to NEET}

NEETs may reflect the impossibility for young people to attain the requirements of normative adult models. In this regard, there are gender specificities as well as personal characteristics to be kept into account. Young girls show a more rapid transition to adulthood than young men (Malmberg and Trempała 1997). In addition, while for young men the consequences of becoming NEET may be mainly related to difficulties in the labor market, young women would also experience negative psychological outcomes (Bynner and Parsons 2002). Education is a second relevant factor associated with NEET. Using data from the British Cohort Study, it was found that low instruction levels are likely to be associated with the NEET rate (Bynner and Parsons 2002). In the same cohort of young people it was shown how uncertain occupational aspirations would be related to NEET (Yates et al. 2011).

Health and mental health conditions may also be associated with the NEET status. For instance, actively searching for a job could be hampered by chronic disorders such as suffering from chronic stress and fatigue (Lim et al. 2016). In terms of mental health, long-term unemployment and loss of interest may lead NEETs to a perception of generalized reduced self-efficacy, which implies resignation and inactivity (Jerusalem and Mittag 1995). At the same time NEETs may experience adverse psychological outcomes, like decreasing of self-estimate or self-efficacy (Heckhausen 2006), and the very impossibility of actively choosing a different direction in life. If prolonged, the NEET state could also determine in the young generations a condition of protracted inertia and social disengagement (Maguire 2015).

Many psychological factors have to be discussed in relation to NEET. First of all, motivation (Dietrich and Salmela-Aro 2013), which is a key factor to favor the transition from school to work (Symonds et al. 2019). Motivation is strictly related to young people's educational aspirations (Hegna 2014), future expectations (Iovu et al. 2018), and investing in active efforts in goal pursuit (Lechner et al. 2016), which are important determinant of educational choices and outcomes. The motivational factor is a determinant of job search intensity, which is a very important variable in terms of the possibility of changing the NEET status (Vanoverberghe et al. 2008).

Motivation is a primary psychological dimension but should be studied in conjunction with other components that, if activated, may protect the individual from becoming NEET. In this regard, three important factors documented in the literature are given by goal attainability, i.e., the ability of self-monitoring one own's objectives 
(Nurmi et al. 2002), the capacity of attributing priorities (Brannen and Nilsen 2002), and intrapersonal agency (Graaf and Zenderen 2013).

Two classical concepts of general psychology, which have to be considered in connection to NEET and youth unemployment are coping styles and the locus of control. The coping style and the ability to cope with stress are a protective factor in terms of completing education and starting a job (Ngai et al. 2014). The internal locus of control is the capacity of the individual to attribute internally the effect of behavior/conduct, which may play a role in relation to NEET and other developmental tasks of adolescence and of the emerging adulthood (Sharon 2016). Career preparation, a factor related to the locus of control, predicts adolescents' satisfaction and career success (Hirschi 2010). However, having an internal locus of control seems a not sufficient condition for protecting young people against becoming NEET for those living in disadvantaged socio-economic conditions (Ng-Knight and Schoon 2017; Vancea and Utzet 2018).

\subsubsection{Familial and peer-group related factors}

At a micro level, parental educational level as well as parents' support may have a protective effect on the risk of becoming NEET, for both male and female teenagers (Alfieri et al. 2015). NEETs are likely to live in a family with one parent, and where parents are not working (Barham et al. 2009). The literature has shown how being NEET is associated with presence of poor or inadequate familial models and backgrounds (Robson and Team 2008), or to intergenerational influences (Bynner and Parsons 2002). At a macro level, being NEET is more frequent in Southern than in Northern European countries, with the former being characterized by a familialistic protective welfare (Esping-Andersen 1990). As concerns the role of the group of peers, it is certainly a fundamental component of socialization in adolescent development, whose effect can go beyond familial factor (Harris 1995). There is also recent literature linking the role of the group of peers with high school dropout (Ripamonti 2018). The role of the peer group and a constellation of negative behaviors, like crime and violence, has been recently highlighted in Canadian NEETs (Henderson et al. 2017), while in Europe this specific issue, to our knowledge, has been less studied.

\subsubsection{Organizational and work-related factors}

There are some factors associated with the NEET status that are related to the organizational and work environment. As to the school environment, recent research from Nordic countries indicates that the excess risk for NEET can be attributed to teenagers' poor performance at school (Berlin et al. 2020). While becoming NEET does not coincide with dropping out from high school, non-completion of school and entering the labor market without qualifications is a strong determinant of transitioning through the NEET condition, especially for women (Salvà-Mut et al. 2016). Basic school achievements, in terms of literacy and numeracy skills, are a consistent protective factor with respect to the possibility of becoming NEET (Barth et al. 2019; Kelly et al. 2012). School is not only a matter of contents or competence, but should actively help teenagers in finding their vocational specificity, which is an important factor in terms of job placement (Vogtenhuber 2014).

There is a large amount of studies documenting the important role of internships, extra-curricular activities, investment on soft skills, and experiences like the "gap year" in helping teenagers in the phase of transition from school to work (Aguilar et al. 2018; Sortheix et al. 2013; Stehlik 2010; Vanoverberghe et al. 2008). The literature has underlined the importance of the psychological dimension of these paths, which should be perceived by the individual as an opportunity to ameliorate his/her own outcome in the labor market (Taylor et al. 2015). Important macro-level variables should be kept into account, since, at least in the EU countries, there is a certain variation in the real possibilities for young people to invest in apprenticeships and internships (Lehmann et al. 2015).

A possible solution to contrast the NEET phenomenon is given by starting a self-employed activity. While, as documented in a large study involving $11 \mathrm{EU}$ countries, this may protect young people from social exclusion and unemployment (Dvoulety et al. 2018), it could be not achievable or realistic for the most frail and inactive segment of NEETs. In fact, given the personal and psychological vulnerability of NEETs, it may be not easy for them, even with the help of formative programs or mentors, to develop a specific business idea (Nabi et al. 2015).

\subsubsection{Contextual factors}

The level of poverty measured at community level has been proposed as a key variable to understand the mechanism of social exclusion, indirectly favoring outcomes like becoming NEET or school dropout (Harding 2003). However, while associated with low socio-economic status, the NEET condition is not exclusive of young people living in poverty or homeless (Thompson 2011). Indeed, being NEET may not be per se a matter of economic disadvantage, but a signal of a developmental difficulty of these teenagers in terms of adjusting their life trajectory in the direction of adulthood (Chen 2011). A role of the geographical context (living in rural and provincial territories vs. cities and metropolises) has also been suggested, at least in terms of school completion (Swanson 
2004). Becoming NEET could be a consequence of the increasing complexity of the EU labor market system (Raffo and Reeves 2000). The presence and prevalence of NEETs in the EU may also be linked to the special needs of the local economy (e.g., request of low-skilled, low-paid workers) (Simmons 2008). At a local level, in an analysis conducted in the Austrian federal states, Bacher et al. reported that expenditures for active labor market policy and other contextual factors can explain the geographical difference in the NEETing rate (Bacher et al. 2017).

\subsubsection{Is NEET a choice?}

Rarely, and for a very low percentage of the young population, becoming NEET has been described as a choice, on a temporary or voluntary basis. These conditions include, for instance, the situation of temporary NEET in which are teenage parents taking care of their children (Contini et al. 2019), which should not be automatically deemed as negative (Simmons and Thompson 2011). Hence, not all NEETs would be at the same risk of social exclusion (Yates and Payne 2006). We remark that voluntary NEETs are counted in the official definition of NEET and contribute to the uncertainty and heterogeneity of the description of this phenomenon.

\subsection{Country setting: Italy}

In Italy the rate of NEETs, considering the age range $15-29$ years, was at $23.4 \%$ in 2018 , with huge regional differences (ISTAT 2019): $15.6 \%$ in Northern provinces, $19.6 \%$ in Central provinces, and $33.8 \%$ in Southern provinces (see the Additional file 1). It is worth putting the investigation of the effect of economic/cultural capital on NEET in the Italian context, which is characterized by relevant geographical differences across the country in terms of both economic performance and welfare state. While the ISTAT has long highlighted the different NEET rate between Northern and Southern provinces, there is not extensive literature addressing the possible determinants of this gap, which is generally and primarily attributed to the territorial differences in the economic performance. In addition, Italian provinces have different history, cultural practices, and civic traditions (Putnam et al. 1994). Italy, like France and other Southern European countries (Ferrera 1996), is characterized by a strong welfare system provided by the State. However, with the regionalization process following the 2001 Constitutional reform, a rather marked variability in welfare regimes across Italian regions did emerge, especially as concerns the organization of vocational training.

Italy is also a country with a highly regulated labor market and, at least until 1997 (the first time-point for the assessment of economic capital in the present investigation) with scarce development of innovative policies connecting formative tracks with the possibility of finding a job, which may have reflected in high unemployment rates in the young generations (Breen 2005). Three labor market reforms (Treu in 1997; Biagi in 2003; Poletti in 2014) have been approved in order to liberalize temporary jobs and to encourage companies hiring new employees. However, the side effect of these Jobs Acts is that the labor market has been made precarious, especially for young people (Barbieri and Scherer 2009). As a consequence, enterprises have been discouraged from investing in permanent training and specialization (Blanchard et al. 2017). This may have led to the high NEET rate that has been described in Italy in the last $10-15$ years.

\subsection{Cultural capital and education}

The dynamics underpinning a complex phenomenon like NEET, reflecting scarce accumulation of human capital, should be investigated also keeping into account the putative role of macro-level variables, such as the economic capital, the social capital, and the cultural capital, as well as their interaction with the micro level (Billari 2004). To provide an example, not being employed or in a formative track could also be related to high-level variables like living in familialistic cultures typical of Southern Europe, which in general provide strong support and protection to teenagers when they are not participating in the labor market (Kalmijn and Saraceno 2008).

Following the Weberian tradition, individuals do not only belong to a certain social class (mainly reflecting their position in the labor market), but also have a certain status in the society, which reflects participation to a collectivity and is not just a constrained function of social class. In this context, the construct of cultural capital (Bourdieu 1986, 1984, 1970) refers to intellectual and educational factors that could foster social mobility, beyond mere financial security or economic assets (economic capital), and beyond relation, group membership, support (social capital). In this paper we adopt the construct of cultural capital on a larger scale than that originally proposed by Bourdieu, i.e., we measure the cultural capital developed in a certain geographical region at an aggregated level (DiMaggio 1982). Indeed cultural capital can be conceived at a macro-system level (Ford and Lerner 1992), in terms of the possibility offered to the population to participate and being involved in the society and in cultural activities (DiMaggio and Mohr 1985). Bourdieu primarily described cultural capital at an individual or familiar level, as a factor contributing to the social reproduction at a macro-level, in terms, for instance, of intraclass difference; this has been operationalized also in the recent literature on educational 
outcomes (Tramonte and Willms 2010). There is justification in the sociological literature to embrace a larger view of cultural capital from a multilevel perspective (Reay 2004). This line of thinking can be attributed to Bourdieu himself (Brubaker 1985), who differentiated between embodied, objectified, and institutionalized cultural capital (Bourdieu 1984), and to the sociologist Paul DiMaggio, who clearly distinguished between cultural capital at an individual level, and cultural capital at a macro level, in terms, for instance, of cultural goods produced by a society (DiMaggio 1991). Other authors have already adopted the construct of cultural capital at an aggregate level and from a systemic perspective, for instance in the ecological literature (Berkes and Folke 1994), or in the economic literature in terms of cultural assets (Throsby 1999). In order to put cultural capital in the context of the present investigation, it is worth remarking that it has been shown how, in Italy, this would be very heterogeneous across provinces (Ripamonti and Barberis 2018). Central provinces would attain the highest levels of cultural capital, followed by Northern and Southern provinces.

\subsection{Economic capital and education}

The economic capital, at different levels of aggregation, is an important predictor of educational attainment. Classical studies of developmental psychology and economics of education have documented that, in children, poverty and family income are associated with cognitive development and behavioral patterns (Duncan et al. 1994). The human capital (Becker 1962) of parents, which is a proxy of the economic opportunities for the family members, has been described as a protective factor for pupils in terms of school achievement and better social outcomes (Brooks-Gunn et al. 2006; Janosz et al. 1997). Quality of home and of day care environments, which are strongly correlated to the economic capital at an aggregate level, have also been described to be associated with cognitive abilities and language development (Broberg et al. 1997; Burchinal et al. 1996). Together, these findings highlight the importance of assessing an adverse outcome such as becoming NEET, keeping into account the specific background of the place where teenagers live, especially in terms of the economic performance. Indeed, the literature has shown that the NEET phenomenon is intrinsically related to the structural economic and labor market characteristics. In a study conducted in Ireland, it was clearly demonstrated the connection of the economic recession of 2008 with the NEET rate, despite the general characteristics of this population were basically the same of the pre-crisis society (Kelly and McGuinness 2015). In this line, European statistical institutes have long underlined that the NEET rate is higher in countries with difficulties in the economic performance (e.g., Italy, Greece or Spain) than Germany or Nordic countries. Moreover, in Southern European countries, like Italy, there is an inter-regional gradient with Northern regions outperforming Southern regions in terms of both the economic performance and the NEET rate (ISTAT 2019). While the association between the economic capital and the NEET rate has been reported at a descriptive level, the structural mechanisms underlying these phenomena are far from being clarified.

\subsection{Hypotheses}

This paper aims to study putative protective factors on the NEET rate through a large-scale and place-based approach, analyzing the role of macro level variables. We pointed to clarify, at a detailed geographical level, the impact of both economic and cultural capital on becoming NEET. A diachronic perspective was employed, assessing cultural capital, economic capital and the NEET rate at three different time points. First, we hypothesized that to high levels of cultural capital in a certain geographical area may correspond valuable cultural opportunities offered to teenagers, and this might promote the development of their cognitive and non-cognitive skills (Heckman and Rubinstein 2001), thus protecting them from becoming NEETs. This prediction also follows from Ripamonti and Barberis (2018), who reported a protective effect of cultural capital on high school dropout, but with relevant differences across Italian provinces. Second, we assumed that living in areas characterized by strong economic performance could have a protective effect on the NEET rate, and we aim to clarify whether such putative effect is of direct type or may be mediated by cultural capital. Third, we aimed to investigate possible mediation effects of cultural/economic capital on the NEET phenomenon.

\section{Methods}

\subsection{Databases}

Data on the NEET rate (at aggregated level) from 103 Italian provinces were obtained from the database of the "Rapporto BES" of the Istituto Nazionale di Statistica (ISTAT 2019). This is an integrated and comprehensive data source on the performance of the Italian provinces that presents the main economic, social and environmental indicators of the country. In particular, the NEET indicator is the measure annually provided by the Institute in the Labor Force Survey. From the ISTAT database we also selected variables on the economic performance. Data on cultural capital have been obtained from the researches on the Quality of Life in Italy yearly published by Il Sole 24 Ore, namely the leading and most authoritative Italian economic newspaper. This database, which also adopts 
some of the ISTAT indicators, has been already described elsewhere (Ripamonti and Barberis 2018).

\subsection{Measures and latent variables}

We considered the ISTAT time-point indicator for NEET in both 2009 and 2017. The indicator refers to the percentage of people aged 15-29 years. who are neither employed nor were in an education or training path, considering the total population in the same age range. As concerns cultural capital, we adopted a multidimensional perspective, taking into account different measures that could be linked to the development of cognitive and non-cognitive skills in young people (Ripamonti and Barberis 2018). For the first time-point (1997), we considered the following indicators: (i) the number of bookshops (BOO97); (ii) the number of art, cultural, and free time associations (ASS97); (iii) the number of cinemas (CIN97). Measures for cultural capital in 2007 were: (i) the percentage of book sale index (BOO07); (ii) the number of art, cultural and free time associations (ASS07); (iii) the number of cinemas (CIN07). These measures are expressed with reference to a target population of 100,000 inhabitants. Analyses were repeated (and shown in the Additional file 1) inserting in the measurement model for cultural capital (iv) the average expenses pro capite to participate in sport events in 1997 (SPO97); the sport index in 2007 (SPO07). We inserted a sport indicator in the operationalization of cultural capital, as this could represent a proxy of the possibilities offered to students, in a certain geographical area, to develop their non-cognitive skills (Ripamonti and Barberis 2018). Since results were very similar, herein we will present the more parsimonious model without adopting the sport indicator.

As to the economic performance, we used measures and proxies of wealth (income, added value, number of enterprises) and of employment adopted, from the ISTAT database, in the researches on the Quality of Life. In particular, in 1997 the indicators were: (i) the income pro capite (INC97); (ii) the number of enterprises for every 100,000 inhabitants (ENT97); (iii) the ratio between job seekers and labor force, i.e., the unemployment rate, which is transformed in the researches on the Quality of life in an employment score for each province (the higher the better performance) (EMP97). In 2007, we adopted the following indicators: (i) the added-value pro capite (INC07); (ii) the number of enterprises for every 100,000 inhabitants (ENT07); (iii) the ratio between job seekers and labor force, as a proxy of unemployment,

\footnotetext{
${ }^{2}$ For illustration see: https://st.ilsole24ore.com/includes2007/speciali/qualitadella-vita/scheda_indice_9.shtml (only available in Italian); see also Ripamonti and Barberis (2018).
}

which is also transformed in the researches on the Quality of life in an employment score (EMP07). ${ }^{2}$ Four latent variables have been introduced, namely CUL97 and CUL07, reflecting the cultural capital of a province in 1997 and 2007; ECO97 and ECO07, describing the economic performance of the Italian provinces in 1997 and 2007, respectively. We collected information on four time-points (1997 and 2007 for the assessment of the cultural/economic capital indicators; 2009 or 2017 for the outcome) since we aimed to disentangle short-run and medium-run (Blanchard et al. 2017) effects of economic/ cultural capital on the NEET rate.

\subsection{Data analysis}

\subsubsection{Descriptive and spatial indicators}

We calculated descriptive indicators (1st and 3rd quartiles, median, interquartile range, $\min$ and $\max$ ) for the variables under study. We used choropleth maps to attain a complete spatial view of the values taken by the statistical units at a detailed geographical level. These maps were also used taking into account the Local Moran index (Moran 1948) in order to study spatial dependency and local peculiarities (or cluster). The Local Moran index belongs to a large class of statistical indicators known in the literature as Local Indicators of Spatial Association (LISA) (Anselin 1995) and provides estimates of spatial autocorrelation disaggregated to the level of the statistical units.

\subsubsection{Structural equation modeling}

We calculated confirmatory Structural Equation Models (SEM) using Covariance Structure Analysis (Jöreskog 1970) through Generalized Least Squares estimation. Models were defined adopting the Linear Structural Equation Modeling (LISREL) terminology and notation. The relation between latent and manifest variables was of reflective type (Loehlin 1987); all analyses were conducted on standardized variables. In the phase of model building, we took into account the values of the Akaike Information Criterion (AIC). Two types of modification indices have been considered: (i) Lagrange multipliers (LM, parameters having the largest LM indices would most increase the model fit); (ii) Wald statistics (in order to eliminate the parameters that are not significant and may be removed without virtually affecting the model fit). As to the error terms matrices, we initially constrained to zero all the extra-diagonal terms, and subsequently we unconstrained only those terms highlighted by LMs and theoretically meaningful. We considered the following indicators of model assessment (Hu and Bentler 1999): (i) the ratio between the value of the Chi square statistic and its degrees of freedom; (ii) the General Fit Index (GFI); (iii) the Adjusted General Fit Index (AGFI); (iv) the Root 
Mean Square Residual (RMR); (v) the Root Mean Square Error of Approximation (RMSEA).

\subsubsection{Mediation analysis}

To evaluate how the effect of cultural capital and of economic capital on NEET occurs in practice, we conducted a mediation analysis. Given the relatively low amount of literature on the interplay between cultural capital, economic capital, and NEET, we adopted an exploratory perspective in the mediation models. Thus, we considered both the possibility that the economic capital could mediate a historical effect of the cultural capital, and vice versa. First, we estimated whether the economic capital could mediate the association between cultural capital and NEET. Second, we estimated whether the cultural capital could mediate the association between economic capital and NEET. Taking economic capital in 2007 (ECO07) as a putative mediator, we aimed to disentangle the total effect of cultural capital in 1997 (CUL97) on NEET in 2009 (NEE09) into a direct effect and an indirect effect that goes through the mediation of the economic capital. This analysis was conducted using the product method (Baron and Kenny 1986), which is based on a decomposition of the total effect in a direct and indirect component, by calculating a regression model for the effect of CUL97 on NEE09 while adjusting for $\mathrm{ECO} 07$, and another regression model assessing the effect of CUL97 on ECO07. Thus, the indirect effect expressing the mediation component can be calculated by taking the effect of CUL97 on ECO07 multiplied by the effect of ECO07 on NEE09. We repeated the same analysis targeting cultural capital in 2007 (CUL07) as a putative mediator of the association between economic capital in 1997 (ECO97) and NEET in 2009 (NEE09). Finally, we re-estimated both mediation models inserting NEE17 as an outcome measure.

\subsubsection{Sensitivity analysis}

SEM work under the assumption of no unmeasured confounding. To explore the possible impact of endogeneity on our findings, we developed a delimited simulation study. We inserted into the SEM previously outlined (with the NEET rate in 2009 as outcome, NEE09) a putative unmeasured confounder, obtained by simulating a vector of values from a Normal r.v. mildly $(\rho=0.30)$, moderately $(\rho=0.60)$ or highly ( $\rho$ $=0.80$ ) correlated to both ECO07 and NEE09. We reestimated the models and we compared the coefficients with those obtained in the original models. A second sensitivity analysis was run to assess the robustness of the choice of the three time points in the SEM models. In particular, we re-ran the same models already explained in the "Structural Equation Modeling" subsection, but inserting the NEET rate after 1 year (2008,
NEE08) or after 5 years (2012, NEE12) from the last assessment of cultural and economic capital (2007). A third sensitivity analysis was performed to explore possible differential effects attributable to the economic capital, which contains two indicators of wealth (income/added value pro-capite and number of enterprises) and one indicator of employment. The same analyses already presented in the "Structural Equation Modeling" subsection have been re-run, firstly by inserting in the economic capital indicator only the GDP/added value component, and secondly by inserting only the employment component.

\section{Results}

\subsection{Descriptive and spatial analysis}

From 2004 to 2018 the NEET rate increased in all Italian macro areas, but particularly in the Southern regions and in the islands (Fig. 2). In addition, as indicated by the box plots shown in Fig. 2, in Southern regions and in the islands, data were much more scattered from the median value than in Northern and Central regions.

Choropleth maps showed an increasing trend between Northern and Southern provinces in terms of the NEET rate (Fig. 3). As indicated by the values of the Local Moran index, in Southern provinces and in the two islands the levels of NEET are generally high and well clustered. Positive spatial autocorrelation has been found also in Northern-East provinces, but with associated much lower rates of NEETs than in Southern or Central provinces.

\subsection{Structural equations modeling}

In Fig. 4 we show the three time-points model adopted to assess the effect of cultural capital and economic capital on the NEET rate. We firstly considered as an outcome measure the prevalence of NEET in 2009 (Model 1); subsequently we repeated the same analysis, but we inserted as outcome variable the prevalence of NEETs in 2017 (Model 2). We tested these models in all the Italian provinces, and afterwards inserting only Northern, Central, or Southern provinces.

Estimating Model 1 for the entire sample of the Italian provinces, we obtained a fit value $\chi^{2} / d f=2.50$ (see Table 1).

The effect of the economic capital in 2007 (ECO07) on the NEET rate in 2009 (NEE09) was significant considering all the Italian provinces $(\beta=-0.81(0.12), t=-6.75, p<0.0001)$, and the Central provinces $(\beta=-0.28(0.11), t=-2.54, p=0.008)$. In the sample of all the Italian provinces, we also found an effect of the cultural capital in 1997 (CUL97) on the economic capital in 2007 (ECO07) ( $\beta=0.36(0.07), t=4.88, p<0.0001)$ and of the 


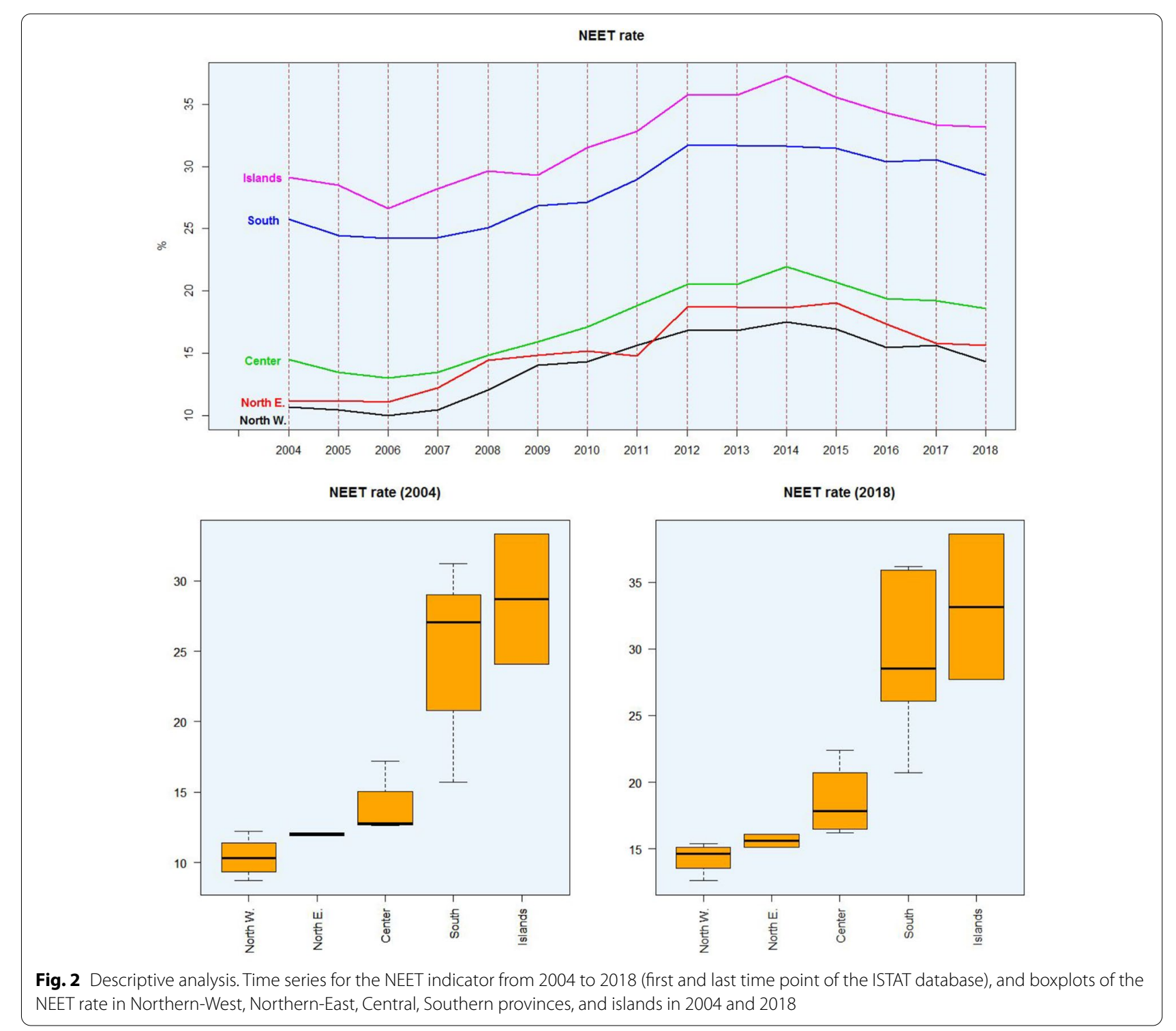

economic capital in 1997 (ECO97) on the cultural capital in 2007 (CUL07) ( $\beta=0.22(0.05), t=4.18, p<0.0001$ ); these results were replicated considering the subsamples of Northern, Central, and Southern provinces. However, we did not obtain any significant finding as concerns the effect from cultural capital in 2007 (CUL07) to the NEET rate in 2009 (NEE09).

Estimating Model 2 for the entire sample of the Italian provinces, it was attained a fit value $\chi^{2} / d f=1.35$ (see Table 2). The relation from the economic capital in 2007 (ECO07) to the NEET rate in 2017 (NEE17) was significant in the entire sample of Italian provinces ( $\beta=-0.94(0.11), t=-8.54, p<0.0001)$, in the Central $(\beta=-0.98(0.19), t=-5.15, p<0.0001)$ and in the Southern provinces $(\beta=-0.81(0.24), t=-3.37, p=0.0008)$, but not in the Northern provinces. Again, we found a significant effect of the cultural capital in 1997 (CUL97) on the economic capital in 2007 (ECO07) ( $\beta=0.45(0.08), t=5.49, p<0.0001)$, and of the economic capital in 1997 (ECO97) on the cultural capital in 2007 (CUL07) $(\beta=0.16(0.04), t=3.49, p=0.0005)$ in all the Italian provinces; analogous patterns did emerge in the subsamples of Northern, Central, and Southern provinces. The relation between the cultural capital in 2007 (CUL07) and the NEET rate in 2017 (NEE17) was never significant. Factor loadings for the measurement model are shown in Table 2.

\subsection{Mediation analysis}

From SEM it emerged a protective effect of the economic capital on the NEET rate. To better understand 


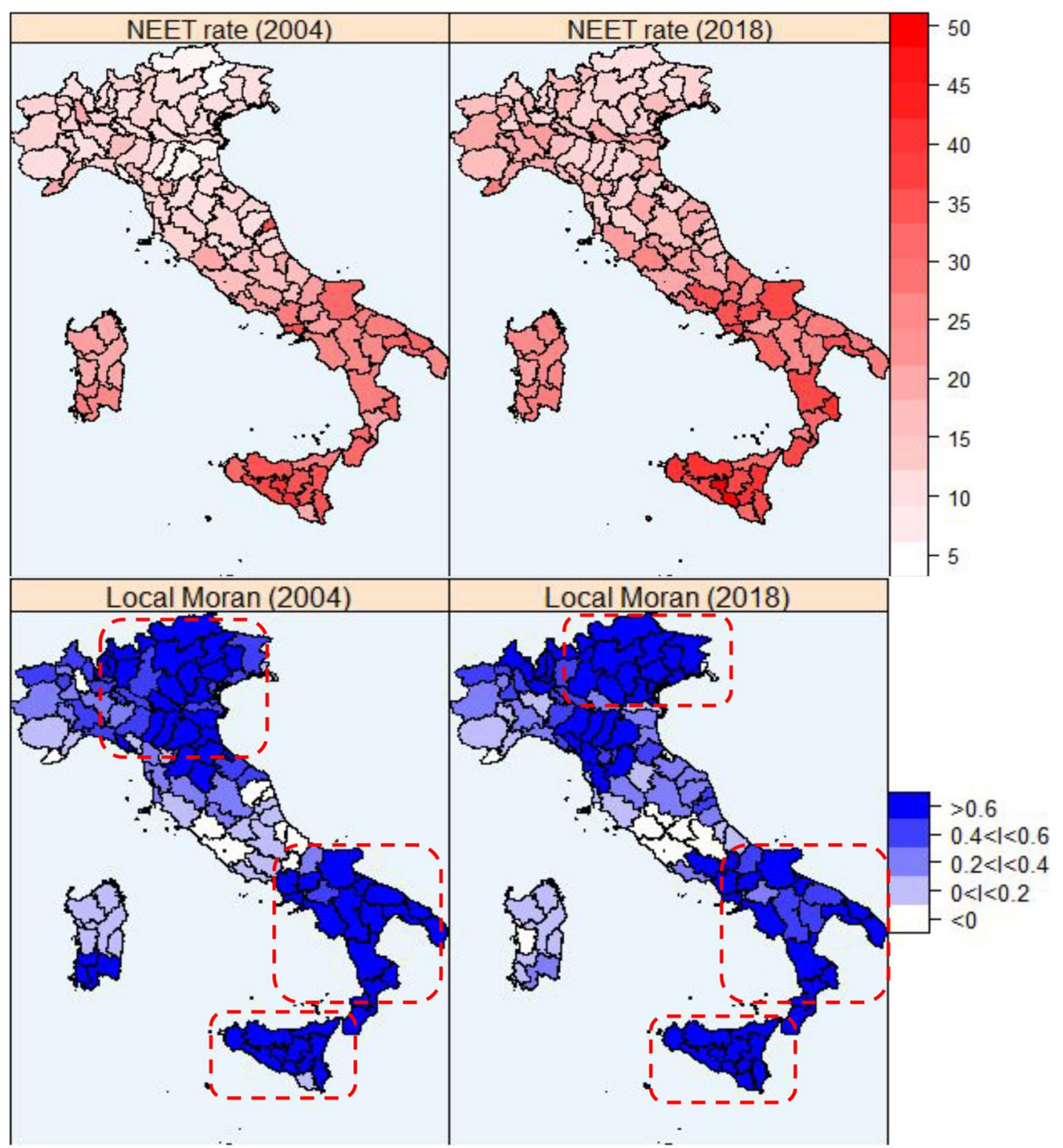

Fig. 3 Spatial analysis. Distribution (above) and Local Moran index of autocorrelation (below) for the NEET rate in Italy in 2004 and 2018

how this effect occurs and the possible interplay of economic capital with cultural capital we conducted a mediation analysis using the product method. After having verified the absence of interaction between the cultural capital in 1997 (CUL97) and the economic capital in 2007 (ECO07), ${ }^{3}$ we inserted the variable economic capital in 2007 (ECO07) as a putative mediator of the effect between cultural capital in 1997 (CUL97) and the NEET rate in 2009 (NEE09) (CUL97 -> ECO07 $->$ NEE09). The direct effect from the cultural capital in 1997 (CUL97) to the NEET rate in 2009 (NEE09) was

\footnotetext{
${ }^{3}$ Creating the quartiles of the CUL97 and ECO07 scores and inserting them as covariates in a multiple regression model with NEE09 as outcome measure.
}

not significant, and for the $83 \%(\mathrm{Z}=7.70, p<0.0001)$, it was attributable to the mediation component played by the economic capital in 2007 (ECO07). We calculated another mediation model (having verified the same assumption of absence of interaction effects), inserting the cultural capital in 2007 (CUL07) as a putative mediator of the effect between the economic capital in 1997 (ECO97) and the NEET rate in 2009 (NEE09) (ECO97 ->CUL07 -> NEE09). Results pointed to $24 \%$ $(\mathrm{Z}=3.17, p=0.001)$ of the effect due to the mediation component of the cultural capital in 2007 (CUL07), and the direct effect from the economic capital in 1997 (ECO97) to the NEET rate in 2009 (NEE09) was also significant $\quad(\beta=-0.36(0.06), Z=-5.52, p<0.0001)$. We repeated the same calculations inserting the NEET 


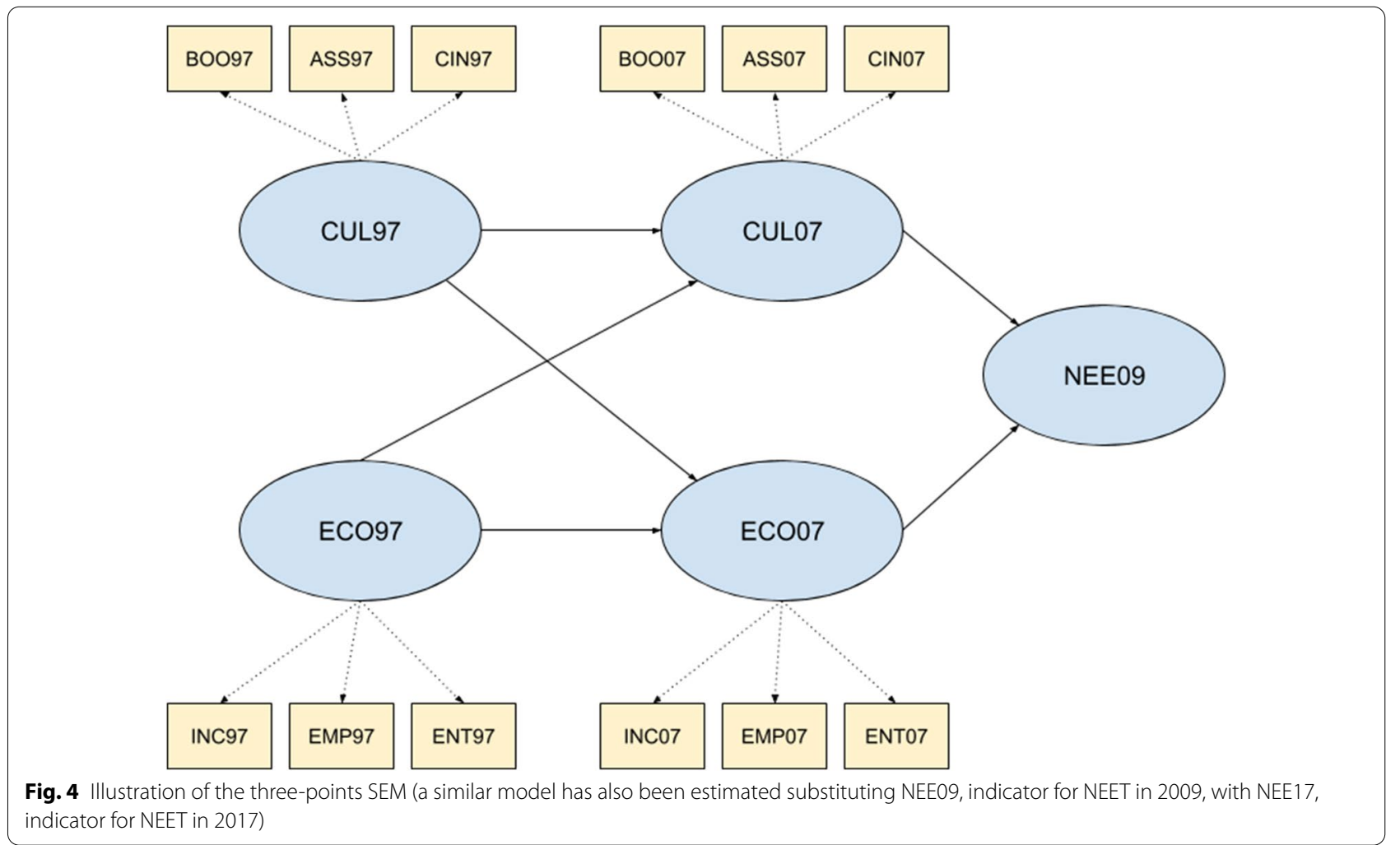

Table 1 Fit index for SEM considering all the Italian provinces (IT), and, separately, Northern (N), Central (C) and Southern (S) provinces

\begin{tabular}{llllll}
\hline Model & $\boldsymbol{\chi}^{\mathbf{2}} \boldsymbol{d} \boldsymbol{d f}$ & GFI & AGFI & RMR & RMSEA \\
\hline Model 1, IT & 2.50 & 0.83 & 0.65 & 0.18 & 0.12 \\
Model 1, N & 1.33 & 0.47 & 0.66 & 0.08 & 0.09 \\
Model 1, C & 1.09 & 0.76 & 0.52 & 0.42 & 0.05 \\
Model 1, S & 1.24 & 0.73 & 0.44 & 0.22 & 0.08 \\
Model 2, IT & 1.35 & 0.48 & 0.66 & 0.11 & 0.09 \\
Model 2, N & 0.96 & 0.47 & 0.64 & 0.22 & 0.01 \\
Model 2, C & 3.28 & 0.38 & 0.32 & 1.15 & 0.26 \\
Model 2, S & 2.07 & 0.75 & 0.50 & 0.08 & 0.18 \\
\hline
\end{tabular}

GFI: Global Fit Index; AGFI: Adjusted Global Fit Index; RMR: Root Mean Square Residual: RMSEA: Root Mean Square Error of Approximation

rate in 2017 (NEE17) as an outcome variable, and we found that the economic capital in 2007 (ECO07) possibly mediated almost the $100 \%(Z=7.32, p<0.0001)$ of the effect of the cultural capital in 1997 (CUL97), while the direct effect of the cultural capital in 1997 (CUL97) on the NEET rate in 2017 (NEE17) was not significant. The variable cultural capital in 2007 (CUL07) mediated about the $16 \%(\mathrm{Z}=2.42, p=0.01)$ of the effect of the economic capital in 1997 (ECO97), and the direct effect from the economic capital in 1997 (ECO97) to the NEET rate in 2017 (NEE17) was also significant ( $\beta=-0.41(0.07), Z=-5.91, p<0.0001)$. To sum up, cultural capital seems to play a weak (and statistically non-significant) role on the NEET rate, and such relation seems strongly mediated by the economic capital. By contrast, in the medium term the economic capital would play a consistent effect on the NEET rate, and such effect seems partly mediated by the cultural capital.

\subsection{Sensitivity analysis}

We conducted a first sensitivity analysis, as described in the Methods section, to assess the possible impact of endogeneity on our models. In this regard we simulated (from Normal random variables), the presence of an unmeasured confounder in the SEM including all the Italian provinces. Three scenarios have been evaluated, inserting a mild, moderate, or strong confounder, respectively. Results (see Table 3) indicate that both the relations from the economic capital in 2007 to the NEET rate in 2009 (ECO07 -> NEE09) and from the economic capital in 2007 to the NEET rate in 2017 (ECO07 - > NEE17) are robust with respect to the presence of a mild or of a moderate unmeasured confounder, but the statistical significance may not be confirmed in case of a strong confounder. While 
Table 2 Factor loadings for the measurement model in all the Italian provinces. We report the estimate (SE)

\begin{tabular}{lll}
\hline & CUL97 & ECO97 \\
\hline BOO97 & $0.79(0.05)^{*}$ & $0.68(0.07)^{*}$ \\
ASS97 & $0.95(0.04)^{*}$ & $1.16(0.06)^{*}$ \\
CIN97 & & $0.36(0.11)^{*}$ \\
INC97 & & $0.99(0.05)^{*}$ \\
EMP97 & & ECO07 \\
ENT97 & CUL07 & $0.96(0.01)^{*}$ \\
\hline BOO07 & $1.05(0.22)^{*}$ & $0.91(0.02)^{*}$ \\
ASS07 & $0.95(0.20)^{*}$ & $0.92(0.02)^{*}$ \\
CIN07 & $0.77(0.18)^{*}$ & \\
INC07 & & \\
EMP07 & & \\
ENT07 & & \\
\hline
\end{tabular}

* Indicates a significant $\mathrm{p}$-value

Table 3 Sensitivity analysis, examining the robustness of the relations ECO07-> NEE09 and ECO07->NEE17, when inserting a mild, moderate or strong unmeasured confounder (UC) in the model

\begin{tabular}{lllc}
\hline & $\boldsymbol{\beta}(\mathrm{SE})$ & $\mathbf{t}$ & $\mathbf{p}$-value \\
\hline ECO07-> NEE09 & & & \\
Original & $-0.81(0.12)$ & -6.75 & $<0.0001$ \\
With mild UC & $-0.89(0.16)$ & -5.45 & $<0.0001$ \\
With moderate UC & $-0.97(0.22)$ & -4.40 & $<0.0001$ \\
With strong UC & $-0.00009(0.00007)$ & -1.33 & 0.18 \\
ECO07-> NEE17 & & & \\
Original & $-0.94(0.11)$ & -8.54 & $<0.0001$ \\
With mild UC & $-0.96(0.14)$ & -6.53 & $<0.0001$ \\
With moderate UC & $-1.07(0.23)$ & -4.50 & $<0.0001$ \\
With strong UC & $-0.00002(0.0007)$ & -0.09 & 0.92 \\
\hline
\end{tabular}

these results are worth reporting, the presence of a strong unmeasured confounder, i.e., a variable related to both the economic capital in 2007 (ECO07) and the NEET rate in 2009/2017 NEE09/NEE17 (with $\rho \geq 0.80$ ) should be justified theoretically and proven empirically.

In a second sensitivity analysis we re-estimated the SEM model for all the Italian provinces, inserting as outcome variable the NEET rate in 2008 (NEE08) or the NEET rate in 2012 (NEE12). We obtained very similar results to those already presented in the "Structural equations modeling" subsection. In particular, the relation from the economic capital in 2007 to the NEET rate in 2008 (ECO07 - > NEE08) was still significant $\quad(\beta=-0.97(0.06), t=-15.14, p<0.0001)$ as well as the relation from the economic capital in 2007 to the NEET rate in 2012 (ECO07 - > NEE12) $(\beta=-1.14(0.13), t=-8.49, p<0.0001)$.

A third sensitivity analysis was run including in the operationalization of the economic capital only the indicators of wealth (GDP/added value), without the employment index. While the result was still in the expected direction, neither the relation from the economic capital in 2007 to the NEET rate in 2009 (ECO07 $->$ NEE09) $\quad(\beta=-0.35(0.87), t=-0.41, p=0.68)$ nor the relation from the economic capital in 2007 to the NEET rate in 2017 (ECO07 $->$ NEE17) $(\beta=-0.40(0.42), t=-0.96, p=0.33) \quad$ proved statistically significant. A similar finding emerged when inserting only the indicator of the employment in the general population without the indicators of wealth; the relationship was negative but not significant, neither in the short run (from the economic capital in 2007 to the NEET rate in 2009, ECO07 -> NEE09: $\quad \beta=-0.32(0.27), t=-1.16, p=0.24)$ nor in the medium run (from the economic capital in 2007 to the NEET rate in 2017, ECO07 - > NEE17: $\beta=-0.01(0.53), t=-0.02, p=0.98)$. Together, these results indicate the importance of considering in the measurement model describing the economic capital both the indicators of wealth and of employment in the general population.

\section{Discussion}

In contemporary European societies the new generations experience an unexpected condition of decline of their status and economic possibilities as compared with the previous generation. One of the phenomena testifying 
this dependence position is represented by NEETs, who are a very heterogeneous group of teenagers and young people with marked and persistent difficulties in the transition from school to the labor market. For an improved organization and implementation of policies contrasting the NEET phenomenon it is important a deep understanding of the determinants of this outcome, including the macro level antecedents. In this paper, we analyzed at a detailed geographical level the relation among cultural capital, economic capital, and the NEET rate. The analyses have been conducted in Italy, which is one of the EU countries with the highest levels of NEETs, and also presents with some structural weaknesses in its vocational system (Ballarino 2015), e.g., lack of investment on the dual apprenticeship system, which is a protective factor against NEET (Eurofound 2012).

Results of the SEMs presented herein point to a protective effect of economic capital on the NEET rate in the short run (2 years), considering all the Italian provinces and the Central provinces. The same protective effect was found in the medium run (10 years) considering all the Italian provinces, the Central and the Southern provinces. In addition, the economic capital strongly mediates the effect of the cultural capital, which, as a direct effect, is not significant. The cultural capital, by itself, does not play a direct protective role on the NEET rate, but may mediate the effect of the economic capital. Our results are in line with the hypothesis that the economic capital, at a macro level, affects the educational and labor market outcomes (Kelly and McGuinness 2015). In addition, as shown in the sensitivity analysis, both components of the economic capital considered in our analyses, i.e., wealth and unemployment, are associated with the NEET rate. Thus, data presented herein clearly point to an increased NEET rate in territories characterized by scarce economic performance. Our findings are in line with the previous literature, which highlights a correlation of the NEET rate with the general unemployment rate and with the GDP level (Eurofound 2012). This paper adds to previous studies a clear timing of the effects, as well as an analysis of the role of economic capital (where both the components of wealth and general unemployment rate have to be kept into account) at a detailed geographical level.

It was a hypothesis of this article that of a putative protective effect of cultural capital on NEET, following a similar finding already described for high school dropout (Ripamonti and Barberis 2018). Differently from such assumption, our findings did not indicate a direct protective effect of cultural capital on the NEET rate. The discrepancy of results emerged herein with those reported in Ripamonti and Barberis (2018) could be explained in terms of the different outcome considered in the two studies. While non-completion of high school is a proxy of attained level of education, becoming NEET may indicate a virtually stable (at least in the short run) vulnerability condition more deeply rooted than that implied by dropping out of high school. It should also be added that in the present study and in Ripamonti and Barberis (2018), following the definitions provided by Public Authorities and Statistical Institutes, two different age cohorts were considered (15-24 for school dropout, including both early school leavers and non-completers of high school; 15-29 for NEET), which could also explain part of the heterogeneity in the results.

An alternative possible explanation to the absence of direct effect of cultural capital on the NEET rate may rely on the definition of NEET provided by the ISTAT and by other national statistical agencies, i.e., the percentage of people aged 15-29 (or 15-34) that are neither on a formative track nor employed. This definition potentially includes both unemployed young people who are nonstudying and are actively searching a job (as in the definition of unemployment rate) and young people who are not studying and are also out of the labor market (inactive non-students). We may hypothesize that cultural capital can affect NEETs capacity to actively search for a job. Indeed, these young people could be motivated to find a job or a formative activity by experiencing an animate cultural environment.

The mediation analysis also showed that, while there does not seem to be a consistent direct protective effect of cultural capital on the NEET rate, such relation is mediated by the economic capital. Thus, a province with high levels of cultural capital would longitudinally increase the likelihood to ameliorate its economic capital, and this would reflect in a better outcome in terms of the NEET rate, both in the short and in the medium run.

Relevant geographical differences emerged in our analyses are also to be highlighted. Considering the subsample of Northern provinces, contrary to our prediction, we did not identify any protective effect of the economic or cultural capital, neither in the short run nor in the medium run. High levels of both economic and cultural capital characterize these provinces. Thus, a possible explanation of these negative results may be in terms of a ceiling effect, i.e., an excellent performance with very low variation in a prediction variable, which may lead to hamper the possibility of capturing a putative protective effect in the regression models. It is also worth observing that the Local Moran index indicates the presence of a certain heterogeneity, despite low values in magnitude, of the NEET indicator in Northern-East provinces, but not in the NorthernWest provinces.

Another form of capital that could be linked to the NEET rate is given by social capital, which, following the 
sociological tradition, could be operationalized in terms of connections, social networks, trust (Coleman 1988). While it would be worth investigating social capital as a putative protective factor for NEET, it is also clearly differentiated from the construct of cultural capital, as originally underlined by Bourdieu (1994). Thus, introducing social capital in this analysis would raise a quite different research question, which we leave open for future research. Social capital could also help to better understand the NEET phenomenon in the Northern provinces, where no significant effect of cultural or economic capital did emerge.

In terms of timing of the effects, in this article we basically adopted an econometric viewpoint. In the economic theory (Blanchard et al. 2017) short-term effects are those described in one or few years, while mediumterm effects generally imply a timing of about a decade. Both cultural and economic capital have been measured before the great recession of 2008. While it is interesting to observe that economic capital plays a protective effect on NEET on both the short run and the medium run (but no effect has been described for cultural capital), results should be carefully interpreted. Indeed, our analysis has only 4 time points. We did not conduct a time-lagged analysis with multiple time points, neither we considered change scores of the NEET rate. These possibilities are open for future investigation using a more comprehensive data source as well as other longitudinal methods for data analysis, such as econometric panels. It is important to remark that in Southern provinces the protective effect of the economic capital on the NEET rate was obtained only in the medium run scenario (i.e., after 10 years), but not in the short run scenario (i.e., after 2 years). These provinces are characterized by low levels of economic performance and by marked spatial heterogeneity in the economic performance (Ripamonti and Barberis 2018), which, together, could imply that the beneficial effect of a slow economic development on the young generation may be detectable only after a decade or longer.

A first limitation of this study is that of having only investigated the potential protective factors on the NEET rate at a macro-variable level, without studying the effect of the macro level on the micro level, in different cultural contexts (Rogoff 2003). In Italy, as well as in other EU countries, large administrative databases on phenomena like NEET, at individual level, are still under construction, and are not currently available on a large scale. This problem also hampers the possibility to properly address the heterogeneity issue in the NEET population. Heterogeneity represents one of the main problems of using the NEET category (Furlong 2006; Yates and Payne 2006) since it probably reflects the presence, under the same umbrella term ("NEET"), of young people with very different backgrounds and developmental histories. Hence it may become difficult to program proper and individualized policies to deal with this phenomenon. The other side of the coin is that, thanks to the uncertainty degree underpinning the NEET definition, the NEET rate may allow to capture, at least at an aggregate level of analysis, particularly vulnerable young people without a job but also without the possibility of developing their human capital in proper formative tracks. This frail segment of the youth population would not be captured by the standard unemployment rates (Rosina 2015).

Even though our analysis presents some limitations, we assume that understanding the role played by high-level variables may drive and provide guidance on future research at a micro-level of analysis. At this stage, macro-micro link studies in all areas of research related to transition to adulthood, including becoming NEET, are still scarce (Buchmann and Kriesi 2011). A second limitation is that we restricted our analyses to the assessment of the economic condition before the economic crisis of 2008, which relevantly weakened the economy of Southern European countries. Young people and the youth labor market were disproportionately affected by the consequences of the crisis (Bell and Blanchflower 2011), as recently documented in terms of youth unemployment and employment trajectories in Spain (Verd et al. 2019). An evaluation of the effects of the great recession (2008 and the double-dip, 2012) in terms of the NEET rate, in relation with the economic and cultural capital, could be a specific issue for a future investigation.

In conclusion, we found that the economic capital (considered in both dimensions of wealth and employment), independently of cultural capital and measured before the economic crisis of 2008, may protect young people from becoming NEET. Peculiar geographical and temporal patterns did emerge, with the effect of the economic capital being particularly relevant in Italian Central provinces, and in the Southern provinces only in the medium run scenario. Findings reported herein indicate relationships among variables at a macro level, and should be complemented by future research at an individual level. Our final message to policy makers is that investment in economic development, especially in economic depressed areas like Southern Italy, may have a beneficial effect on the NEET rate in the short and in the medium run. The role of the economic capital may be partly mediated by the cultural capital, which, however, does not seem to play an independent effect on the NEET rate. Investment on culture and education cannot be isolated from investment on economic development, and a multifactorial phenomenon like NEET 
demands complex, differentiated, and multi-component policies.

\section{Supplementary Information}

The online version contains supplementary material available at https://doi. org/10.1186/s12651-021-00296-y.

Additional file 1: Fig. S1. NEET rate (males) for EU(27) countries, and, specifically for France, Germany, Italy and Spain (Eurostat data). Fig. S2. NEET rate (females) for EU(27) countries, and, specifically for France, Germany, Italy and Spain (Eurostat data). Fig. S3. Boxplots of the NEET rate (males) in the European countries $(n=27), 2004-2018$ (Eurostat data). Fig. S4. Boxplots of the NEET rate (females) in the European countries $(n=27)$ 2004-2018 (Eurostat data). Fig. S5. Boxplots of the NEET rate (females vs. males) in the European countries ( $n=27)$, in 2004, 2009, 2018 (ISTAT data).

\section{Acknowledgements}

None.

\section{Authors' contributions}

ER conceived the study, completed data analyses and drafted the manuscript; SB collaborated in the data management, data analysis, and drafting of the manuscript. Both authors read and approved the final manuscript.

\section{Funding}

None.

\section{Availability of data and materials \\ Upon request.}

\section{Declarations}

\section{Competing interests}

The authors declare that they have no competing interests.

\section{Author details}

'Milan Center for Neuroscience, University of Milan-Bicocca, Piazza dell'Ateneo Nuovo 1, 20126 Milan, Italy. ${ }^{2}$ Department of Neurobiology, Care Sciences and Society, Karolinska Institutet, A Medical University, Stockholm, Sweden. ${ }^{3}$ Department of Statistics and Quantitative Methods, University of Milan-Bicocca, Milan, Italy. ${ }^{4}$ Ente Nazionale Per II Microcredito, Rome, Italy.

Received: 25 September 2020 Accepted: 12 April 2021 Published online: 28 April 2021

\section{References}

Aguilar, M.I., Corrales-Herrero, H., Dìaz, B., Garcìa-Crespo, D., Rodrìguez-Prado, B.: Time to first significant job for vocational graduates in Spain. J. Youth Stud. 21, 235-252 (2018)

Alfieri, S., Sironi, E., Marta, E., Rosina, A., Marzana, D.: Young Italian NEETs (Not in Employment, Education, or Training) and the influence of their family background. Eur. J. Psychol. 11, 311 (2015)

Anselin, L.: Local indicators of spatial association—LISA. Geogr. Anal. 27, 93-115 (1995)

Arnett, J.J.: Emerging adulthood in Europe: A response to Bynner. J. Youth Stud. 9. 111-123 (2006)

Autor, D.H., Katz, L.F., Kearney, M.S.: Trends in US wage inequality: Revising the revisionists. Rev. Econ. Stat. 90, 300-323 (2008)

Bacher, J., Koblbauer, C., Leitgöb, H., Tamesberger, D.: Small differences matter: how regional distinctions in educational and labour market policy account for heterogeneity in NEET rates. J. Labour Mark. Res. 51, 4 (2017)

Ballarino, G.: School in contemporary Italy: structural features and current policies. In: Ascoli, U., Pavolini, E. (eds.) The Italian Welfare State in a
European Perspective: A Comparative Analysis, pp. 181-208. Policy Press, Bristol (2015)

Barbieri, P., Scherer, S.: Labour market flexibilization and its consequences in Italy. Eur. Sociol. Rev. 25, 677-692 (2009)

Barham, C., Walling, A., Clancy, G., Hicks, S., Conn, S.: Young people and the labour market. Econ. Labour Mark. Rev. 3, 17-29 (2009)

Baron, R.M., Kenny, D.A.: The moderator-mediator variable distinction in social psychological research: Conceptual, strategic, and statistical considerations. J. Pers. Soc. Psychol. 51, 1173 (1986)

Barro, R.: Economic growth in a cross section of countries. Q. J. Econ. 106, 407-443 (1991)

Barth, E., Keute, A.L., Schøne, P., von Simson, K., Steffensen, K.: NEET status and early versus later skills among young adults: Evidence from linked register-PIAAC data. Scand. J. Educ. Res. 65, 1-13 (2019)

Battistin, E., Rettore, E., Trivellato, U.: Choosing between alternative classification criteria to measure the labour force state. J. R. Stat. Soc. Ser. A (Statistics Soc. 170, 5-27 (2007)

Becker, G.S.: Investment in human capital: A theoretical analysis. J. Polit. Econ 70, 9-49 (1962)

Bell, D.N.F., Blanchflower, D.G.: Young people and the Great Recession. Oxford Rev. Econ. Policy. 27, 241-267 (2011)

Berkes, F., Folke, C.: Investing in cultural capital for sustainable use. In: Koskoff, S. (ed.) Investing in Natural Capital: The Ecological Economics to Sustainability. Centre for Resource Economics. Island Press, Washington (1994)

Berlin, M., Kääriälä, A., Lausten, M., Andersson, G., Brännström, L.: Long-term NEET among young adults with experience of out-of-home care: A comparative study of three Nordic countries Int. J. Soc Welf. (2020). https://doi.org/10.1111/ijsw.12463

Billari, F:: Becoming an adult in Europe: A macro(/micro)-demographic perspective. Demogr. Res. 3, 15-44 (2004)

Blanchard, O., Amighini, A., Giavazzi, F.: Macroeconomics: a European perspective. Pearson Education, London (2017)

Bourdieu, P.: La Reproduction. Editions de Minuit, Paris (1970)

Bourdieu, P.: Distinction. Routledge, London (1984)

Bourdieu, P.: The forms of capital. In: Richardson, J. (ed.) Handbook of theory and research for the sociology of education, pp. 241-258. Greenwood, New York (1986)

Brannen, J., Nilsen, A.: Young people's time perspectives: From youth to adulthood. Sociology 36, 513-537 (2002)

Breen, R.: Explaining cross-national variation in youth unemployment: Market and institutional factors. Eur. Sociol. Rev. 21, 125-134 (2005)

Broberg, A.G., Wessels, H., Lamb, M.E., Hwang, C.P.: Effects of day care on the development of cognitive abilities in 8-year-olds: A longitudinal study. Dev. Psychol. 33, 62 (1997)

Brooks-Gunn, J., Cunha, F., Duncan, G., Heckman, J.J., Sojourner, A.: A Reanalysis of the IHDP Program. Northwestern University, Evanston (2006)

Brubaker, R.: Rethinking classical theory: The sociological vision of Pierre Bourdieu. Theory Soc. 14, 745-775 (1985)

Brzinsky-Fay, C.: Lost in transition? Labour market entry sequences of school leavers in Europe. Eur. Sociol. Rev. 23, 409-422 (2007)

Buchmann, M.C., Kriesi, I.: Transition to adulthood in Europe. Annu. Rev. Sociol. 37, 481-503 (2011)

Burchinal, M.R., Roberts, J.E., Nabors, L.A., Bryant, D.M.: Quality of center child care and infant cognitive and language development. Child Dev. 67, 606-620 (1996)

Bynner, J., Parsons, S.: Social exclusion and the transition from school to work: The case of young people not in education, employment, or training (NEET). J. Vocat. Behav. 60, 289-309 (2002)

Chen, Y.-W.: Once a NEET always a NEET? Experiences of employment and unemployment among youth in a job training programme in Taiwan. Int. J. Soc. Welf. 20, 33-42 (2011)

Coleman, J.S.: Social capital in the creation of human capital. Am. J. Sociol. 94, S95-S120 (1988)

Contini, D., Filandri, M., Pacelli, L.: Persistency in the NEET state: a longitudinal analysis. J. Youth Stud. 22, 959-980 (2019)

de Graaf, W., van Zenderen, K.: School-to-work transition: the interplay between institutional and individual processes. J. Educ. Work. 26 $121-142(2013)$

Dietrich, J., Salmela-Aro, K.: Parental involvement and adolescents' career goal pursuit during the post-school transition. J. Adolesc. 36, 121-128 (2013) 
DiMaggio, P.: Cultural capital and school success: The impact of status culture participation on the grades of US high school students. Am. Sociol. Rev. 47, 189-201 (1982)

DiMaggio, P.: Social structure, institutions, and cultural goods: The case of the US. In: Social theory for a changing society. Boulder: Westview Press (1991)

DiMaggio, P., Mohr, J.: Cultural capital, educational attainment, and marital selection. Am. J. Sociol. 90, 1231-1261 (1985)

Duncan, G.J., Brooks-Gunn, J., Klebanov, P.K.: Economic deprivation and early childhood development. Child Dev. 65, 296-318 (1994)

Dvoulety, O., Mühlböck, M., Warmuth, J., Kittel, B.: 'Scarred'young entrepreneurs. Exploring young adults' transition from former unemployment to self-employment. J. Youth Stud. 21, 1159-1181 (2018)

Elder, S.: What does NEETs mean and why is the concept so easily misinterpreted? International Labour Office, Geneve (2015)

Esping-Andersen, G.: The three worlds of welfare capitalism. Princeton University Press, New York (1990)

Eurofound: NEETs--Young people not in employment, education or training: Characteristics, costs and policy responses in Europe. Publications Office of the European Union, 2012, Luxembourg, Luxembourg City (2012)

Ferrera, M.: The 'Southern model'of welfare in social Europe. J. Eur. Soc. Policy. 6, 17-37 (1996)

Ford, D.H., Lerner, R.M.: Developmental systems theory: An integrative approach. Sage Publications Inc, London (1992)

Furlong, A.: Not a very NEET solution: representing problematic labour market transitions among early school-leavers. Work. Employ. Soc. 20, 553-569 (2006)

Harding, D.J.: Counterfactual models of neighborhood effects: The effect of neighborhood poverty on dropping out and teenage pregnancy. Am. J. Sociol. 109, 676-719 (2003)

Harris, J.R.: Where is the child's environment? A group socialization theory of development. Psychol. Rev. 102, 458 (1995)

Heckhausen, J.: Developmental regulation in adulthood: Age-normative and sociostructural constraints as adaptive challenges. Cambridge University Press, Cambridge (2006)

Heckman, J.J., Rubinstein, Y.: The importance of noncognitive skills: Lessons from the GED testing program. Am. Econ. Rev. 91, 145-149 (2001)

Hegna, K.: Changing educational aspirations in the choice of and transition to post-compulsory schooling-a three-wave longitudinal study of Oslo youth. J. Youth Stud. 17, 592-613 (2014)

Henderson, J.L., Hawke, L.D., Chaim, G., Network, N.Y.S.P.: Not in employment, education or training: Mental health, substance use, and disengagement in a multi-sectoral sample of service-seeking Canadian youth. Child. Youth Serv. Rev. 75, 138-145 (2017)

Hirschi, A.: The role of chance events in the school-to-work transition: The influence of demographic, personality and career development variables. J. Vocat. Behav. 77, 39-49 (2010)

$\mathrm{Hu}, \mathrm{L} .$, Bentler, P.: Cutoff criteria for fit indexes in covariance structure analysis: Conventional criteria versus new alternatives. Struct. Equ. Model. 6, 1-55 (1999)

lovu, M.-B., Huaruaguș, P.-T., Roth, M.: Constructing future expectations in adolescence: relation to individual characteristics and ecological assets in family and friends. Int. J. Adolesc. Youth. 23, 1-10 (2018)

ISTAT: Rapporto BES 2019: Il benessere equo e sostenibile in Italia. Rome (2019)

Janosz, M., LeBlanc, M., Boulerice, B., Tremblay, R.E.: Disentangling the weight of school dropout predictors: A test on two longitudinal samples. J. Youth Adolesc. 26, 733-762 (1997)

Jerusalem, M., Mittag, W.: Self-efficacy in stressful life transitions. In: Bandura, A. (ed.) Self-efficacy in changing societies, pp. 177-201. Cambridge University Press, Cambridge (1995)

Jones, S.R.G., Riddell, W.C.: The measurement of unemployment: An empirical approach. Econometrica. 147-161 (1999)

Jöreskog, K.G.: A general method for analysis of covariance structures. Biometrika 57, 239-251 (1970)

Kalmijn, M., Saraceno, C.: A comparative perspective on intergenerational support: Responsiveness to parental needs in individualistic and familialistic countries. Eur. Soc. 10, 479-508 (2008)

Kelly, E., McGuinness, S.: Impact of the Great Recession on unemployed and NEET individuals' labour market transitions in Ireland. Econ. Syst. 39 59-71 (2015)
Kelly, E., McGuinness, S., O'Connell, P.J.: Transitions to long-term unemployment risk among young people: Evidence from Ireland. J. Youth Stud. 15, 780-801 (2012)

Lechner, C.M., Tomasik, M.J., Silbereisen, R.K.: Preparing for uncertain careers: How youth deal with growing occupational uncertainties before the education-to-work transition. J. Vocat. Behav. 95, 90-101 (2016)

Lehmann, W., Taylor, A., Hamm, Z.:'Go west young man!'Youth apprenticeship and opportunity structures in two Canadian provinces. J. Educ. Work. 28, 44-65 (2015)

Lim, V.K.G., Chen, D., Aw, S.S.Y., Tan, M.: Unemployed and exhausted? Jobsearch fatigue and reemployment quality. J. Vocat. Behav. 92, 68-78 (2016)

Loehlin, J.C.: Latent variable models. Erlbaum, Hillsdale (1987)

Maguire, S.: NEET, unemployed, inactive or unknown-why does it matter? Educ. Res. 57, 121-132 (2015)

Malmberg, L.-E., Trempała, J.: Anticipated transition to adulthood: The effect of educational track, gender, and self-evaluation on Finnish and Polish adolescents' future orientation. J. Youth Adolesc. 26, 517-537 (1997)

Martin, G.: A portrait of the youth labor market in 13 countries, 1980-2007. Mon. Labor Rev. 132, 3-21 (2009)

Mayer, K.U.: The paradox of global social change and national path dependencies: Life course patterns in advanced societies. In: Woodward, A., Kohli, M. (eds.) Inclusions and exclusions in European societies, pp. 103-124. Routledge, London (2004)

Moran, P.A.: The interpretation of statistical maps. J. R. Stat. Soc. Ser. B. 10, 243-251 (1948)

Nabi, G., Walmsley, A., Holden, R.: Pushed or pulled? Exploring the factors underpinning graduate start-ups and non-start-ups. J. Educ. Work. 28, 481-506 (2015)

Narendranathan, W., Elias, P.: Influences of past history on the incidence of youth unemployment: Empirical findings for the UK. Oxf. Bull. Econ. Stat. 55, 161-185 (1993)

Ng-Knight, T., Schoon, I.: Can locus of control compensate for socioeconomic adversity in the transition from school to work? J. Youth Adolesc. 46 2114-2128 (2017)

Ngai, S.S.Y., Cheung, J.C.-K., To, S., Luan, H., Zhao, R.: Economic disadvantage and transitional outcomes: a study of young people from low-income families in Hong Kong. Int. J. Adolesc. Youth. 19, 318-335 (2014)

Nurmi, J.-E., Salmela-Aro, K., Koivisto, P.: Goal importance and related achievement beliefs and emotions during the transition from vocational schoo to work: Antecedents and consequences. J. Vocat. Behav. 60, 241-261 (2002)

Putnam, R.D., Leonardi, R., Nanetti, R.Y.: Making democracy work: Civic traditions in modern Italy. Princeton University Press, Princeton (1994)

Raffo, C., Reeves, M.: Youth transitions and social exclusion: developments in social capital theory. J. Youth Stud. 3, 147-166 (2000)

Reay, D.: Education and cultural capital: The implications of changing trends in education policies. Cult. Trends. 13, 73-86 (2004)

Ripamonti, E.: Risk factors for dropping out of high school: A review of contemporary, international empirical research. Adolesc. Res. Rev. $\mathbf{3}$ 321-338 (2018)

Ripamonti, E., Barberis, S.: The effect of cultural capital on high school dropout: An investigation in the Italian provinces. Soc. Indic. Res. 139, 1257-1279 (2018)

Robson, K., Team, M.C.E.: Becoming NEET in Europe: A comparison of predictors and later-life outcomes. In: Global Network on Inequality MiniConference (2008)

Rogoff, B.: The cultural nature of human development. Oxford University Press, Oxford (2003)

Rosina, A.: NEET. Giovani che non studiano e non lavorano, Vita e pensiero, Milano (2015)

Salvà-Mut, F., Thomás-Vanrell, C., Quintana-Murci, E.: School-to-work transitions in times of crisis: the case of Spanish youth without qualifications. J. Youth Stud. 19, 593-611 (2016)

Sharon, T.: Constructing adulthood: Markers of adulthood and well-being among emerging adults. Emerg. Adulthood. 4, 161-167 (2016)

Simmons, R.: Raising the age of compulsory education in England: A NEET solution? Br. J. Educ. Stud. 56, 420-439 (2008)

Simmons, R., Thompson, R.: Education and training for young people at risk of becoming NEET: findings from an ethnographic study of work-based learning programmes. Educ. Stud. 37, 447-450 (2011) 
Sortheix, F.M., Dietrich, J., Chow, A., Salmela-Aro, K.: The role of career values for work engagement during the transition to working life. J. Vocat. Behav. 83, 466-475 (2013)

Stehlik, T.: Mind the gap: School leaver aspirations and delayed pathways to further and higher education. J. Educ. Work. 23, 363-376 (2010)

Swanson, C.B.: Sketching a portrait of public high school graduation: Who graduates? Who doesn't. In: Orfield, G. (ed.) Dropouts in America: Confronting the graduation rate crisis, pp. 13-40. Harvard Education Press, Cambridge (2004)

Symonds, J., Schoon, I., Eccles, J., Salmela-Aro, K.: The Development of motivation and amotivation to study and work across age-graded transitions in adolescence and young adulthood. J. Youth Adolesc. 48, 1131-1145 (2019)

Taylor, A., Lehmann, W., Raykov, M.: "Should I stay or should I go?" Exploring high school apprentices' pathways. J. Educ. Work. 28, 652-676 (2015)

Thompson, R.: Individualisation and social exclusion: the case of young people not in education, employment or training. Oxford Rev. Educ. 37, 785-802 (2011)

Throsby, D.: Cultural capital. J. Cult. Econ. 23, 3-12 (1999)

Tramonte, L., Willms, J.D.: Cultural capital and its effects on education outcomes. Econ. Educ. Rev. 29, 200-213 (2010)

Vancea, M., Utzet, M.: School-to-work transition: The case of Spanish NEETs. J. Youth Stud. 21, 869-887 (2018)
Vanoverberghe, J., Verhaest, D., Verhofstadt, E., Omey, E.: The transition from school to work in Flanders: a duration analysis. J. Educ. Work. 21, 317-331 (2008)

Verd, J.M., Barranco, O., Bolibar, M.: Youth unemployment and employment trajectories in Spain during the Great Recession: what are the determinants? J. Labour Mark. Res. 53, 4 (2019)

Vogtenhuber, S.:The impact of within country heterogeneity in vocational specificity on initial job matches and job status. J. Vocat. Behav. 85, 374-384 (2014)

Yates, S., Payne, M.: Not so NEET? A critique of the use of 'NEET'in setting targets for interventions with young people. J. Youth Stud. 9, 329-344 (2006)

Yates, S., Harris, A., Sabates, R., Staff, J.: Early occupational aspirations and fractured transitions: a study of entry into 'NEET'status in the UK. J. Soc. Policy. 40, 513-534 (2011)

\section{Publisher's Note}

Springer Nature remains neutral with regard to jurisdictional claims in published maps and institutional affiliations.

\section{Submit your manuscript to a SpringerOpen ${ }^{\circ}$ journal and benefit from:}

- Convenient online submission

- Rigorous peer review

- Open access: articles freely available online

- High visibility within the field

- Retaining the copyright to your article

Submit your next manuscript at $\gg$ springeropen.com 